\title{
Nonlinear Rank-Based Analog Loop Filters in Delta-Sigma Analog-to-Digital Converters for Mitigation of Technogenic Interference
}

\author{
Alexei V. Nikitin \\ Nonlinear Corp. \\ Manhattan, Kansas, USA \\ E-mail: avn@nonlinearcorp.com
}

\author{
Ruslan L. Davidchack \\ Dept, of Mathematics, U. of Leicester \\ Leicester, UK \\ E-mail: rld8@leicester.ac.uk
}

\begin{abstract}
Since at any given frequency a linear filter affects both the noise and the signal of interest proportionally, when a linear filter is used to suppress the interference outside of the passband of interest the resulting signal quality is affected only by the total power and spectral composition, but not by the type of the amplitude distribution of the interfering signal. Thus a linear filter cannot improve the passband signal-to-noise ratio, regardless of the type of noise. On the other hand, a nonlinear filter has the ability to disproportionately affect signals with different temporal and/or amplitude structures, and it may reduce the spectral density of non-Gaussian interferences in the signal passband without significantly affecting the signal of interest. As a result, the signal quality can be improved in excess of that achievable by a linear filter. Such non-Gaussian (and, in particular, impulsive) noise can originate from a multitude of natural and technogenic (man-made) phenomena. The technogenic noise specifically is a ubiquitous and growing source of harmful interference affecting communication and data acquisition systems, and such noise may dominate over the thermal noise. While the non-Gaussian nature of technogenic noise provides an opportunity for its effective mitigation by nonlinear filtering, current state-of-the-art approaches employ such filtering in the digital domain, after analog-to-digital conversion. In the process of such conversion, the signal bandwidth is reduced, which substantially diminishes the effectiveness of the subsequent noise removal techniques. In this paper, we focus on impulsive noise mitigation, and propose to incorporate impulsive noise filtering of the analog input signal into loop filters of $\Delta \Sigma$ analog-todigital converters (ADCs). Such ADCs thus combine analog-todigital conversion with analog nonlinear rank filtering, enabling mitigation of various types of in-band non-Gaussian noise and interference, including broadband impulsive interference. An important property of the presented approach is that, while being nonlinear in general, the proposed ADCs largely behave linearly. They exhibit nonlinear behavior only intermittently, in response to noise outliers, thus avoiding the detrimental effects, such as instabilities and intermodulation distortions, often associated with nonlinear signal processing.

Index Terms - analog signal processing; analog nonlinear filters; analog rank filters; nonlinear differential clippers; $\Delta \Sigma$ modulators; $\Delta \Sigma$ ADCs; electromagnetic interference; technogenic interference; man-made interference; non-Gaussian noise; impulsive noise.
\end{abstract}

\section{INTRODUCTION}

Non-Gaussian (and, in particular, impulsive) noise affecting communication and data acquisition systems can originate from a multitude of natural and technogenic (man-made) phenomena in a variety of applications. Examples of natural impulsive noise sources include ice cracking (in polar regions) and snapping shrimp (in warmer waters) affecting underwater acoustics [1]-[3]. A particular example of non-Gaussian interference is electromagnetic interference (EMI), which is a widely recognized cause of reception problems in communications and navigation devices. The detrimental effects of EMI are broadly acknowledged in the industry and include reduced signal quality to the point of reception failure, increased bit errors which degrade the system and result in lower data rates and decreased reach, and the need to increase power output of the transmitter, which increases its interference with nearby receivers and reduces the battery life of a device. Electrical man-made noise is transmitted into a system through the galvanic (direct electrical contact), electrostatic coupling, electromagnetic induction, or RF waves. Examples of systems and services harmfully affected by technogenic noise include various sensor, communication, and navigation devices and services [4]-[15], wireless internet [16], coherent imaging systems such as synthetic aperture radar [17], cable, DSL and power line communications [18]-[24], wireless sensor networks [25], and many others. An impulsive noise problem also arises when devices based on the ultra-wideband (UWB) technology interfere with narrowband communication systems such as WLAN [26] or CDMA-based cellular systems [27].

A major and rapidly growing source of EMI in communication and navigation receivers is other transmitters that are relatively close in frequency and/or distance to the receivers. Multiple transmitters and receivers are increasingly combined in single devices, which produces mutual interference. A typical example is a smartphone equipped with cellular, WiFi, Bluetooth, and GPS receivers, or a mobile WiFi hotspot containing an HSDPA and/or LTE receiver and a WiFi transmitter operating concurrently in close physical proximity. Other typical sources of strong EMI are on-board digital circuits, clocks, buses, and switching power supplies. This physical proximity, combined with a wide range of possible transmit and receive powers, creates a variety of challenging interference scenarios. Existing empirical evidence [8], [28], 
[29] and its theoretical support [6], [7], [10] show that such interference often manifests itself as impulsive noise, which in some instances may dominate over the thermal noise [5], [8], [28].

The non-Gaussian noise described above affects the input (analog) signal. The current state-of-the-art approach to its mitigation is to convert the analog signal to digital, then apply digital nonlinear filters to remove this noise [30], [31, e.g.]. There are two main problems with this approach. First, in the process of analog-to-digital conversion the signal bandwidth is reduced, and an initially impulsive broadband noise will appear less impulsive [7]-[10], [32]. Thus its removal by digital filters may be much harder to achieve. Second, digital nonlinear filters may not be able to work in real time, as they are typically much more computationally intensive than linear filters. In addition, high-amplitude outliers in the input signal may cause ADC saturation [8, e.g.], making the subsequent noise removal by digital filtering ineffective or outright impossible.

A better approach would be to filter impulsive noise from the analog input signal before the ADC, but such methodology is not widely known, even though the concepts of rank filtering of continuous signals are well understood [32]-[37]. In this paper, we propose to incorporate impulsive noise filtering of the analog input signal into loop filters of delta-sigma $(\Delta \Sigma)$ ADCs.

Nowadays, $\Delta \Sigma$ ADCs are used for converting analog signals over a wide range of frequencies, from DC to several megahertz. These converters comprise a highly oversampling modulator followed by a digital/decimation filter that together produce a high-resolution digital output [38]-[40]. As discussed in Section III, which reviews the basic principle of operation of $\Delta \Sigma$ ADCs from a time domain prospective, a sample of the digital output of a $\Delta \Sigma$ ADC represents its continuous (analog) input by a weighted average over a discrete time interval (that should be smaller than the inverse Nyquist rate) around that sample. When the signal, however, is affected by non-Gaussian noise such as, for example, an outlier (e.g. impulsive or transient) noise and/or interference, other measures of central tendency such as a median or a truncated mean value, or an L-estimator [41]-[43], can better approximate the signal of interest. Following our previously introduced approaches to analog nonlinear filtering [32]-[37], here we outline nonlinear modifications to analog loop filters of $\Delta \Sigma$ ADCs that enable combining analog-to-digital conversion with mitigation of various types of in-band non-Gaussian noise and interference, especially that of technogenic (manmade) origin, including broadband impulsive interference.

\section{Analog RAnK Filters FOR Mitigation OF OUTLIER Noise}

In the simplified illustration that follows, our focus is not on providing precise definitions and rigorous proof of the statements and assumptions, but on outlining the general idea of employing analog nonlinear rank-based filters for mitigation of outlier (impulsive) noise, and thus improving the performance of a communications receiver in the presence of such noise.

\section{A. Motivation and simplified system model}

Let us assume that the input noise affecting a baseband signal of interest with unit power consists of two additive components: (i) a Gaussian component with the power $P_{\mathrm{G}}$ in the signal passband, and (ii) an outlier (impulsive) component with the power $P_{\mathrm{i}}$ in the signal passband. Thus if a linear antialiasing filter is used before the analog-to-digital conversion (ADC), the resulting signal-to-noise ratio (SNR) can be expressed as $\left(P_{\mathrm{G}}+P_{\mathrm{i}}\right)^{-1}$.

For simplicity, let us further assume that the outlier noise is white and consists of short (with the characteristic duration much smaller than the reciprocal of the bandwidth of the signal of interest) random pulses with the average inter-arrival times significantly larger than their duration, yet significantly smaller than the reciprocal of the signal bandwidth. When the bandwidth of such noise is reduced to within the baseband by linear filtering, its distribution will be well approximated by Gaussian [44]. Thus the observed noise in the baseband can be considered Gaussian, and we can use the Shannon formula [45] to calculate the channel capacity.

Let us now assume that we use a nonlinear antialiasing filter such that it behaves linearly, and affects the signal and noise proportionally, when the baseband power of the impulsive noise is smaller than a certain fraction of that of

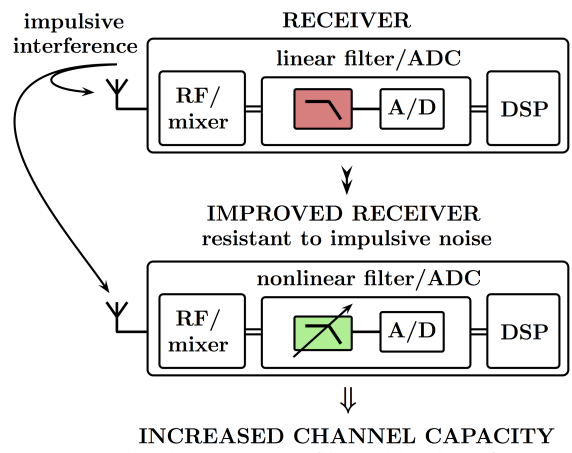

in the presence of impulsive interference

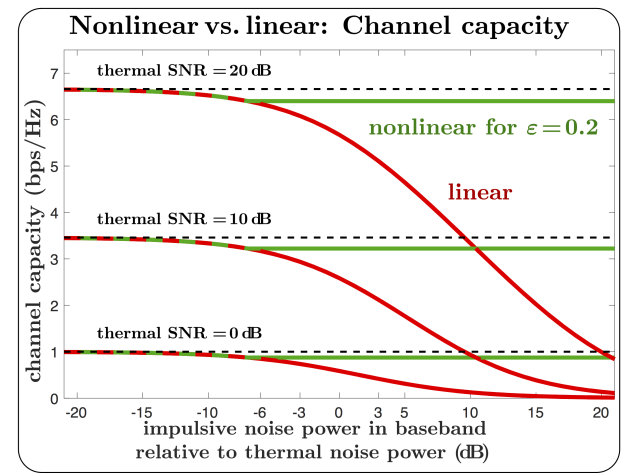

Figure 1. Simplified diagram of improving receiver performance in the presence of impulsive interference. 
the Gaussian component, $P_{\mathrm{i}} \leq \varepsilon P_{\mathrm{G}}(\varepsilon \geq 0)$ resulting in the SNR $\left(P_{\mathrm{G}}+P_{\mathrm{i}}\right)^{-1}$. However, when the baseband power of the impulsive noise increases beyond $\varepsilon P_{\mathrm{G}}$, this filter maintains its linear behavior with respect to the signal and the Gaussian noise component, while limiting the amplitude of the outlier noise in such a way that the contribution of this noise into the baseband remains limited to $\varepsilon P_{\mathrm{G}}<P_{\mathrm{i}}$. Then the resulting baseband SNR will be $\left[(1+\varepsilon) P_{\mathrm{G}}\right]^{-1}>\left(P_{\mathrm{G}}+P_{\mathrm{i}}\right)^{-1}$. We will view the observed noise in the baseband as Gaussian, and use the Shannon formula to calculate the limit on the channel capacity.

As one can see from this example, by disproportionately affecting high-amplitude outlier noise while otherwise preserving linear behavior, such nonlinear antialiasing filter will provide resistance to impulsive interference, limiting the effects of the latter, for small $\varepsilon$, to an insignificant fraction of the Gaussian noise. Fig. 1 illustrates this with a simplified diagram of improving receiver performance in the presence of impulsive interference by employing such analog nonlinear filter before the ADC. In this illustration, $\varepsilon=0.2$.

\section{B. Analog nonlinear filters with the desired behavior}

The analog nonlinear filters with the behavior outlined above can be constructed based on the methodology introduced in [33], which relies on the transformation of discrete or continuous signals into normalized continuous scalar fields with the mathematical properties of distribution functions. This methodology enables a variety of nonlinear signal processing techniques that naturally incorporate the consideration of such distributional properties, including those which have no digital counterparts.

For example, the time-dependent amplitude distribution $\Phi(D, t)$ of a continuous signal $x(t)$ obtained in a time window $w(t)$ can be expressed as

$$
\Phi(D, t)=w(t) * \mathcal{F}_{\Delta D}[D-x(t)],
$$

where $D$ is a threshold value, asterisk denotes convolution, and $\mathcal{F}_{\Delta D}(D)$ is a discriminator function that changes monotonically from 0 to 1 in such a way that most of this change occurs over some characteristic range of threshold values $\Delta D$ around zero. Since $\Phi(D, t)$ can be viewed as a surface in the three-dimensional space $(t, D, \Phi)$, the expression

$$
\Phi\left(D_{q}(t), t\right)=q, \quad 0<q<1,
$$

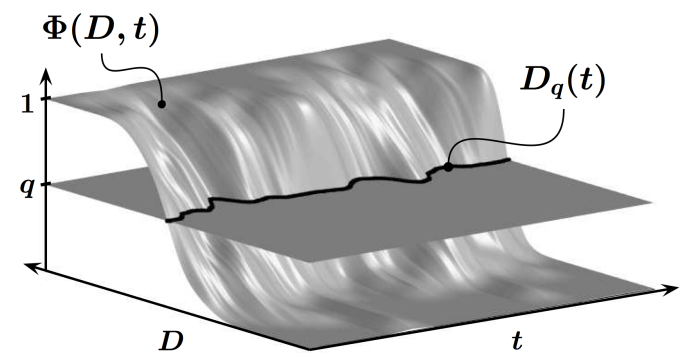

Figure 2. $\quad D_{q}(t)$ as a level curve of the distribution function $\Phi(D, t)$. defines $D_{q}(t)$ as a level (or contour) curve obtained from the intersection of the surface $\Phi=\Phi(D, t)$ with the plane $\Phi=q$, as illustrated in Fig. 2.

An explicit (albeit differential) equation of the level curve $D_{q}(t)$ can be obtained by differentiating equation (2) with respect to time (see, for example, [46], p. 551, eq. (4.29)), leading to

$$
\frac{\mathrm{d} D_{q}}{\mathrm{~d} t}=-\frac{\partial \Phi\left(D_{q}, t\right) / \partial t}{\phi\left(D_{q}, t\right)}+\nu\left[q-\Phi\left(D_{q}, t\right)\right], \quad \nu>0 .
$$

In (3), $\phi(D, t)=\partial \Phi(D, t) / \partial D$ is the amplitude density of $x(t)$ in the time window $w(t)$, and, since $\Phi(D, t)$ is a monotonically increasing function of $D$ for all $t$, the added term in the right-hand side ensures the convergence of the solution to the chosen quantile order $q$ regardless of the initial condition. It can be shown that, depending on the shape of the discriminator function $\mathcal{F}_{\Delta D}(D)$, equation (3) corresponds to a variety of nonlinear filters with desired characteristics. For example, in the limit $\Delta D \rightarrow 0$ equation (3) describes an analog rank filter (e.g. a median filter for $q=1 / 2$, as illustrated below) in an arbitrary time window $w(t)$.

\section{Analog Median Filter}

The digital median filter introduced in the early 1970s [41] is a widely recognized tool for removing outlier (i.e. impulsive) noise. From equation (4.6) in [33], an expression for the output $\chi(t)$ of an "exact" (or "true") analog median filter in an exponential time window with the time constant $\tau_{0}$ can be written as

$$
\dot{\chi}(t)=\lim _{\alpha \rightarrow 0} \frac{\frac{1}{2}-\mathcal{F}_{2 \alpha}[\chi(t)-x(t)]}{\int_{-\infty}^{t} \mathrm{~d} s \exp \left(\frac{s-t}{\tau_{0}}\right) f_{2 \alpha}[\chi(t)-x(s)]},
$$

where $x(t)$ is the input signal, the dot over the variable denotes its time derivative, $\mathcal{F}_{2 \alpha}(x)$ is a discriminator function with a characteristic width $2 \alpha$, and $f_{2 \alpha}(x)=\mathrm{d} \mathcal{F}_{2 \alpha}(x) / \mathrm{d} x$ is its respective probe. In equation (4), functions $\mathcal{F}_{2 \alpha}(x)$ and $f_{2 \alpha}(x)$ are such that $\lim _{\alpha \rightarrow 0} \mathcal{F}_{2 \alpha}(x)=\theta(x)$ is the Heaviside unit step function [47] and $\lim _{\alpha \rightarrow 0} f_{2 \alpha}(x)=\delta(x)$ is the Dirac $\delta$ function [48]. The parameter $\alpha$ can be called the resolution parameter.

\section{1st order Analog Differential Clipper (ADiC)}

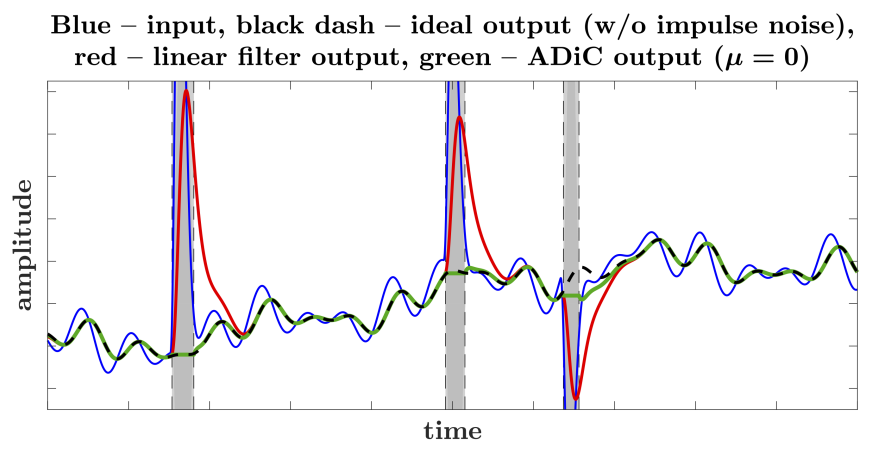

Figure 3. Resistance of $\mathrm{ADiC}$ with $\mu=0$ to outlier noise. The shaded time intervals correspond to nonlinear $\mathrm{ADiC}$ behavior (zero rate of change). 
Let us now consider a particularly simple nonlinear filter that can be expressed as

$$
\dot{\chi}=\frac{x-\chi}{\tau_{0}}\left[\theta(\alpha-|x-\chi|)+\frac{\mu \tau_{0}}{|x-\chi|} \theta(|x-\chi|-\alpha)\right],
$$

where $0 \leq \mu$ is the rate parameter (with units "amplitude per time"). This filter can be derived, after some specific approximations, as a linear combination of those expressed by equations (3) and (4). Note that when $|x-\chi| \leq \alpha$ (e.g., in the limit $\alpha \rightarrow \infty$ ) equation (5) describes a 1st order analog linear lowpass filter (RC integrator). When the magnitude of the difference signal $|x-\chi|$ exceeds the resolution parameter $\alpha$, however, the rate of change of the output is limited to the rate parameter $\mu$ (zero for $\mu=0$ ), and no longer depends on the magnitude of the incoming signal $x(t)$, providing an output insensitive to outliers with a characteristic amplitude determined by the resolution parameter $\alpha$. We shall call the filter described by equation (5) a 1st order Analog Differential Clipper $(A D i C)$. Note that for a sufficiently large $\alpha$ this filter would exhibit nonlinear behavior only intermittently, in response to noise outliers, while otherwise acting as a 1st order linear lowpass filter.

Fig. 3 illustrates resistance of an $\mathrm{ADiC}$ with $\mu=0$ to outlier noise, in comparison with a 1st order linear lowpass filter with the same time constant. The shaded time intervals correspond to nonlinear $\mathrm{ADiC}$ behavior (zero rate of change). Note that the clipping (i.e. zero rate of change of the ADiC output) is performed differentially, based on the magnitude of the difference signal $|x-\chi|$ and not that of the input signal $x$.

Further note that for $\mu=\alpha / \tau_{0}$ equation (5) corresponds to the Canonical Differential Limiter described in [9], [10], [24], [32], and in the limit $\alpha \rightarrow 0$ it corresponds to the Median Tracking Filter described in Section VI-A.

\section{TIME DOMAIN ANALYSIS OF 1ST- AND 2ND-ORDER $\Delta \boldsymbol{\Sigma}$ ADCS WITH LINEAR ANALOG LOOP FILTERS}

Nonlinear filters do not have well-defined impulse and frequency responses, and frequency domain representation is of limited use in the analysis of their behavior. Thus let us first describe the basic $\Delta \Sigma$ ADCs with 1st- and 2nd-order linear analog loop filters in the time domain. Such 1st- and 2nd-order $\Delta \Sigma$ ADCs are illustrated in panels I and II of Fig. 4, respectively. Note that the vertical scales of the shown fragments of the signal traces vary for different fragments.

We assume that if the input $D$ to the flip-flop is greater than zero, $D>0$, at a specific instance in the clock cycle (e.g. the rising edge), then the output $\bar{Q}$ takes a negative value $\bar{Q}=-V_{c}$. If $D<0$ at a rising edge of the clock, then the output $\bar{Q}$ takes a positive value $\bar{Q}=V_{c}$. At other times, the output $\bar{Q}$ does not change. We also assume in this example that $x(t)$ is effectively band-limited, and is bounded by $V_{c}$ so that $|x(t)|<V_{c}$ for all $t$. Further, the clock frequency $F_{\mathrm{s}}$ is significantly higher (e.g. by more than about 2 orders of magnitude) than the bandwidth $B_{x}$ of $x(t), \log _{10}\left(F_{\mathrm{s}} / B_{x}\right) \gtrsim 2$. It can be then shown that, with the above assumptions, the input $D$ to the flip-flop will be a zero-mean signal with an average zero crossing rate much higher than the bandwidth of $x(t)$.

Note that in the limit of infinitely large clock frequency $F_{\mathrm{s}}$ $\left(F_{\mathrm{S}} \rightarrow \infty\right)$ the behavior of the flip-flop would be equivalent to that of an analog comparator. Thus, while in practice a finite flip-flop clock frequency is used, based on the fact that it is orders of magnitude larger that the bandwidth of the signal of interest we may use continuous-time (e.g. $(w * y)(t)$ and $x(t-$ $\Delta t)$ ) rather than discrete-time (e.g. $(w * y)[k]$ and $x[k-m])$ notations in reference to the ADC outputs, as a shorthand to simplify the mathematical description of our approach.

As can be seen in Fig. 4, for the 1st-order modulator shown in panel I

$$
\overline{x(t)-y(t)}=0,
$$

and for the 2nd-order modulator shown in panel II

$$
\frac{\mathrm{d}}{\mathrm{d} t} \overline{y(t)}=\frac{1}{\tau}[\overline{x(t)-y(t)}],
$$

where the overlines denote averaging over a time interval between any pair of threshold (including zero) crossings of $D$ (such as, e.g., the interval $\Delta T$ shown in Fig. 4). Indeed, for a continuous function $f(t)$, the time derivative of its average
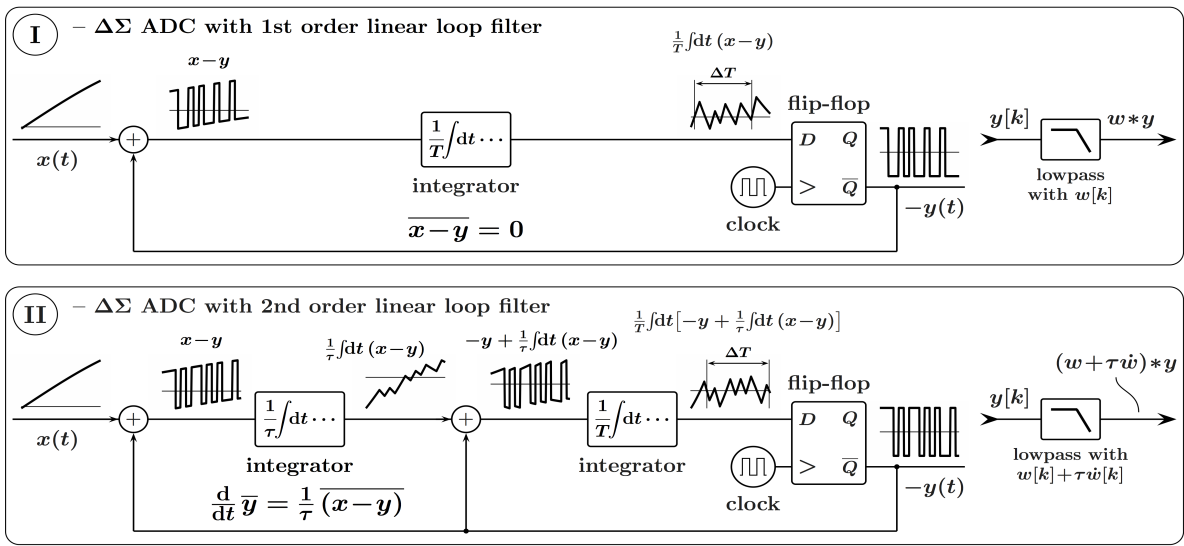

Figure 4. $\Delta \Sigma$ ADCs with 1st-order (I) and 2nd-order (II) linear loop filters. 
over a time interval $\Delta T$ can be expressed as

$$
\frac{\mathrm{d}}{\mathrm{d} t} \overline{f(t)}=\frac{\mathrm{d}}{\mathrm{d} t}\left[\frac{1}{\Delta T} \int_{t-\Delta T}^{t} \mathrm{~d} s f(s)\right]=\frac{1}{\Delta T}[f(t)-f(t-\Delta T)],
$$

and will be zero if $f(t)-f(t-\Delta T)=0$.

Now, if the time averaging is performed by a lowpass filter with an impulse response $w(t)$ and a bandwidth $B_{w}$ much smaller than the clock frequency, $B_{w} \ll F_{\mathrm{s}}$, equation (6) implies that the filtered output of the 1st-order $\Delta \Sigma$ ADC will be effectively equal to the filtered input,

$$
(w * y)(t)=(w * x)(t)+\delta y,
$$

where the term $\delta y$ (the "ripple", or "digitization noise") is small and will further be neglected. We will assume from here on that the filter $w(t)$ has a flat frequency response and a constant group delay $\Delta t$ over the bandwidth of $x(t)$. Then equation (9) can be rewritten as

$$
(w * y)(t)=x(t-\Delta t),
$$

and the filtered output will accurately represent the input signal.

Since $y(t)$ is a two-level staircase signal with a discrete step duration $n / F_{\mathrm{s}}$, where $n$ is a natural (counting) number, it can be accurately represented by a 1-bit discrete sequence $y[k]$ with the sampling rate $F_{\mathrm{s}}$. Thus the subsequent conversion to the discrete (digital) domain representation of $x(t)$ (including the convolution of $y[k]$ with $w[k]$ and decimation to reduce the sampling rate) is rather straightforward and will not be discussed further.

If the input to a 1st-order $\Delta \Sigma$ ADC consists of a signal of interest $x(t)$ and an additive noise $\nu(t)$, then the filtered output can be written as

$$
(w * y)(t)=x(t-\Delta t)+(w * \nu)(t),
$$

provided that $|x(t-\Delta t)+(w * \nu)(t)|<V_{c}$ for all $t$. Since $w(t)$ has a flat frequency response over the bandwidth of $x(t)$, it does not change the power spectral density of the additive noise $\nu(t)$ in the signal passband, and the only improvement in the passband signal-to-noise ratio for the output $(w * y)(t)$ would come from the reduction of the quantization noise $\delta y$ by a well designed filter $w(t)$.

Similarly, equation (7) implies that the filtered output of the 2nd-order $\Delta \Sigma$ ADC will be effectively equal to the filtered input further filtered by a 1st order lowpass filter with the time constant $\tau$ and the impulse response $h_{\tau}(t)$,

$$
(w * y)(t)=\left(h_{\tau} * w * x\right)(t) .
$$

From the differential equation for a 1st order lowpass filter it follows that $h_{\tau} *(w+\tau \dot{w})=w$, and thus we can rewrite equation (12) as

$$
\left(h_{\tau} *(w+\tau \dot{w}) * y\right)(t)=\left(h_{\tau} * w * x\right)(t) .
$$

Provided that $\tau$ is sufficiently small (e.g., $\tau \lesssim 1 /\left(4 \pi B_{x}\right)$ ), equation (13) can be further rewritten as

$$
((w+\tau \dot{w}) * y)(t)=(w * x)(t)=x(t-\Delta t) .
$$

The effect of the 2nd-order loop filter on the quantization noise $\delta y$ is outside the scope of this paper and will not be discussed.

\section{IV. $\Delta \boldsymbol{\Sigma}$ ADC WITH CMTF-BASED LOOP FILTER}

Let us now consider the modifications to a 2nd-order $\Delta \Sigma$ ADC depicted in Fig. 5. (Note that the vertical scales of the shown fragments of the signal traces vary for different fragments.) We will assume from here on that the 1st order lowpass filters with the time constant $\tau$ and the impulse response $h_{\tau}(t)$ shown in the figure have a bandwidth (as signified by its $3 \mathrm{~dB}$ corner frequency) that is much larger than the bandwidth of the signal of interest $B_{x}$, yet much smaller than the sampling (clock) frequency $F_{\mathrm{s}}$. For example, the bandwidth of $h_{\tau}(t)$ can be approximately equal to the geometric mean of $B_{x}$ and $F_{\mathrm{s}}$, resulting in the following value for $\tau$ :

$$
\tau \approx \frac{1}{2 \pi \sqrt{B_{x} F_{\mathrm{s}}}} .
$$

As one can see in Fig. 5, the first integrator (with the time constant $\gamma \tau$ ) is preceded by a blanker, where the blanking function $\mathcal{B}_{\alpha}(x)$ is defined as

$$
\mathcal{B}_{\alpha}(x)=\left\{\begin{array}{cc}
x & \text { for }|x| \leq \alpha \\
0 & \text { otherwise }
\end{array},\right.
$$

and where $\alpha$ is the blanking value.

As shown in the figure, the input $x(t)$ and the output $y(t)$ can be related by

$$
\frac{\mathrm{d}}{\mathrm{d} t} \overline{h_{\tau} * y}=\frac{1}{\gamma \tau} \overline{\mathcal{B}_{\alpha}\left(h_{\tau} *(x-y)\right)},
$$

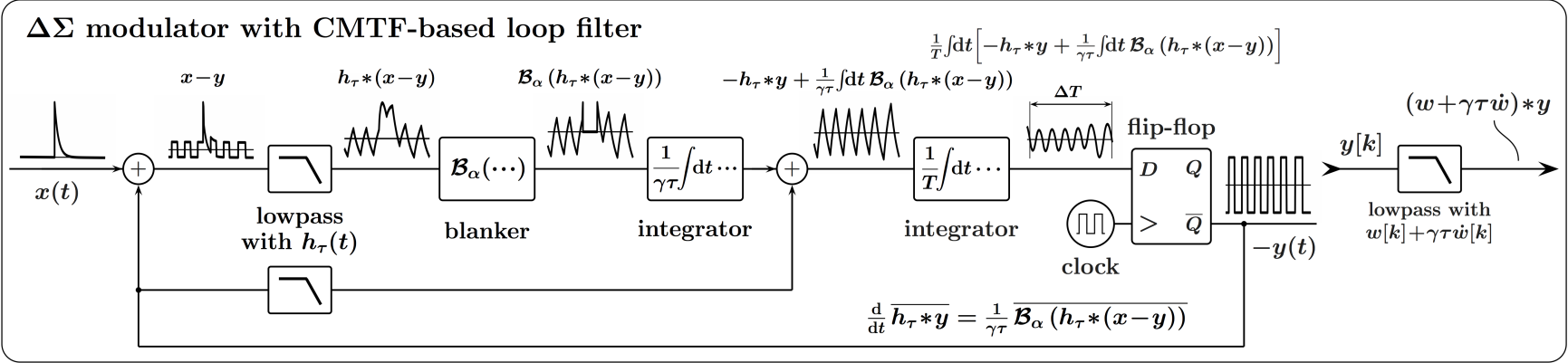

Figure 5. $\Delta \Sigma$ ADC with CMTF-based loop filter. 


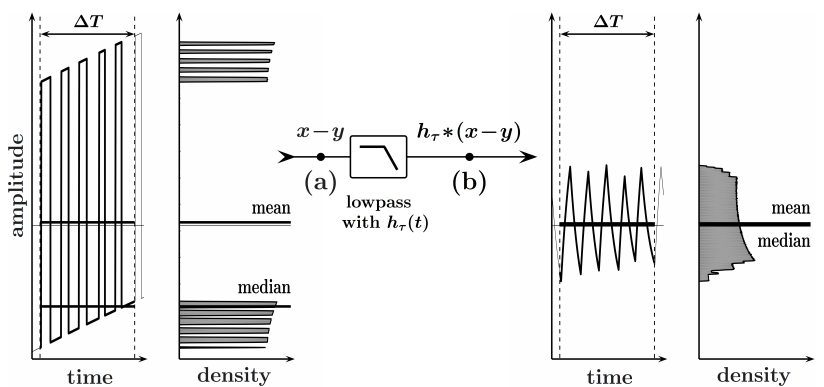

Figure 6. Modifying the amplitude density of the difference signal $x-y$ by a 1 st order lowpass filter.

and the filter represented by equation (17) can be referred to as a Clipped Mean Tracking Filter (CMTF). Note that without the time averaging equation (16) corresponds to the ADiC described by equation (5) with $\mu=0$, where $x$ and $\chi$ replaced by $h_{\tau} * x$ and $h_{\tau} * y$, respectively.

The utility of the 1st order lowpass filters $h_{\tau}(t)$ is, first, to modify the amplitude density of the difference signal $x-y$ so that for a slowly varying signal of interest $x(t)$ the mean and the median values of $h_{\tau} *(x-y)$ in the time interval $\Delta T$ become effectively equivalent, as illustrated in Fig. 6. However, the median value of $h_{\tau} *(x-y)$ is more robust when the narrow-band signal of interest is affected by short-duration outliers such as broadband impulsive noise, since such outliers will not be excessively broadened by the wide-band filter $h_{\tau}(t)$. In addition, while being wide-band, this filter prevents the amplitude of the background noise observed at the input of the blanker from being excessively large.

With $\tau$ given by equation (15), the parameter $\gamma$ may be chosen as

$$
\gamma \approx \frac{1}{4 \pi B_{x} \tau} \approx \frac{1}{2} \sqrt{\frac{F_{\mathrm{s}}}{B_{x}}},
$$

and the relation between the input and the output of the $\Delta \Sigma$ ADCs with a CMTF-based loop filter can be expressed as

$$
x(t-\Delta t) \approx((w+\gamma \tau \dot{w}) * y)(t) .
$$

Note that for large blanking values such that $\alpha \geq\left|h_{\tau} *(x-y)\right|$ for all $t$, according to equation (17) the average rate of change of $h_{\tau} * y$ is proportional to the average of the difference signal $h_{\tau} *(x-y)$. When the magnitude of the difference signal $h_{\tau} *(x-y)$ exceeds the blanking value $\alpha$, however, the average rate of change of $h_{\tau} * y$ is zero and no longer depends on the magnitude of $h_{\tau} * x$, providing an output insensitive to outliers with a characteristic amplitude determined by the blanking value $\alpha$.

Since linear filters are generally better than median for removing broadband Gaussian (e.g. thermal) noise, the blanking value in the CMTF-based topology should be chosen to ensure that the CMTF-based $\Delta \Sigma$ ADC performs effectively linearly when outliers are not present, and that it exhibits nonlinear behavior only intermittently, in response to outlier noise. A robust approach to establishing such a blanking value is outlined in Section VI.

\section{SIMPLIFIED PERFORMANCE EXAMPLE}

Let us first use a simplified synthetic signal to illustrate the essential features of our approach, and the advantages provided by the proposed $\Delta \Sigma$ ADC with the CMTF-based loop filter configuration when the impulsive noise affecting the signal of interest dominates over a low-level background Gaussian noise.

The signal of interest consists of two fragments of two sinusoidal tones with $0.9 V_{c}$ amplitudes, and with frequencies $B_{x}$ and $B_{x} / 8$, respectively, separated by zero-value segments. While pure sine waves are chosen for an ease of visual assessment of the effects of the noise, one may envision that the low-frequency tone corresponds to a vowel in a speech signal, and that the high-frequency tone corresponds to a fricative consonant.

For all $\Delta \Sigma$ ADCs in these examples, the flip-flop clock frequency is $F_{\mathrm{s}}=N B_{x}$, where $N=1024$. For the 2ndorder loop filter in this illustration $\tau=\left(4 \pi B_{x}\right)^{-1}$. The time constant $\tau$ of the 1st order lowpass filters in the CMTF-based loop filter is $\tau=\left(2 \pi B_{x} \sqrt{N}\right)^{-1}=\left(64 \pi B_{x}\right)^{-1}$, and $\gamma=16$ (resulting in $\gamma \tau=\left(4 \pi B_{x}\right)^{-1}$ ). The parameter $\alpha$ is chosen as $\alpha=V_{c}$. The output $y[k]$ of the $\Delta \Sigma$ ADC with the 1 storder linear loop filter (panel I of Fig. 4) is filtered with a digital lowpass filter with the impulse response $w[k]$. The outputs of the $\Delta \Sigma$ ADCs with the 2nd-order linear (panel II of Fig. 4) and the CMTF-based (Fig. 5) loop filters are filtered with a digital lowpass filter with the impulse response $w[k]+\left(4 \pi B_{x}\right)^{-1} \dot{w}[k]$. The impulse and frequency responses of $w[k]$ and $w[k]+\left(4 \pi B_{x}\right)^{-1} \dot{w}[k]$ are shown in Fig. 7.

As shown in panel I of Fig. 8, the signal is affected by a mixture of additive white Gaussian noise (AWGN) and white impulse (outlier) noise components, both band-limited to approximately $B_{x} \sqrt{N}$ bandwidth. As shown in panel II, in the absence of the outlier noise, the performance of all $\Delta \Sigma$ $\mathrm{ADC}$ in this example is effectively equivalent, and the amount of the AWGN is such that the resulting signal-to-noise ratio for
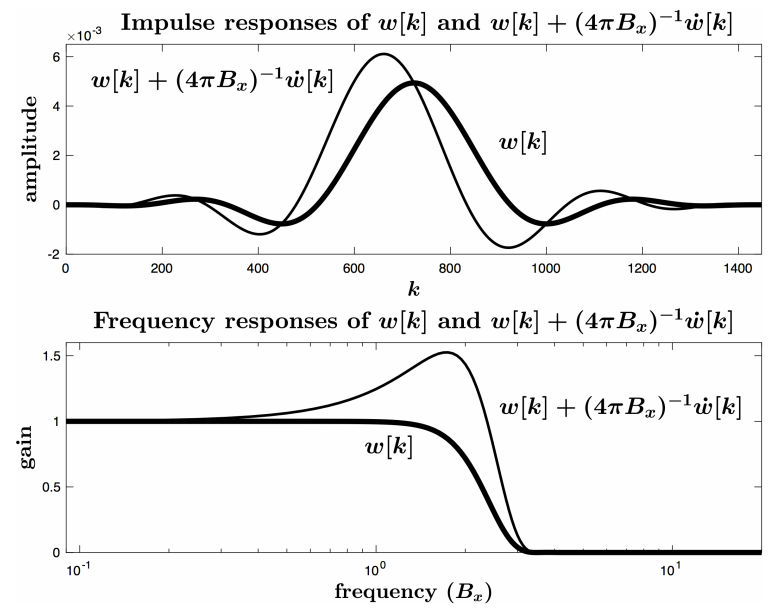

Figure 7. Impulse and frequency responses of $w[k]$ and $w[k]+\left(4 \pi B_{x}\right)^{-1} \dot{w}[k]$ used in the examples of Fig. 8. 


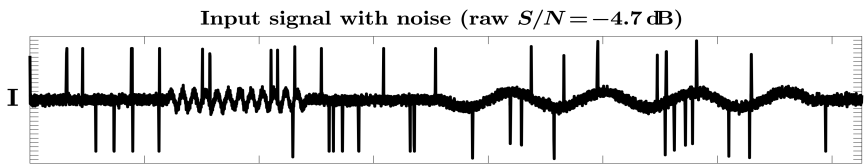

Outputs for all loop filters and AWGN only $(S / N=19.6 \mathrm{~dB})$

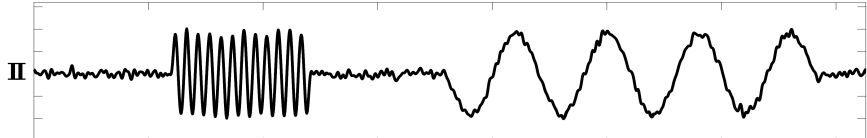

$\Delta \Sigma$ ADC with 1st-order linear loop filter $(S / N=5.8 \mathrm{~dB})$

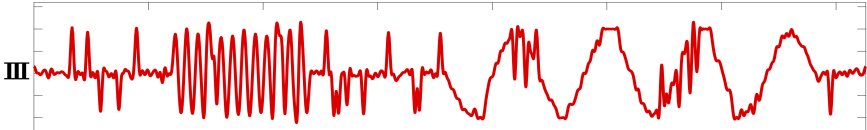

$\Delta \Sigma$ ADC with 2nd-order linear loop filter $(S / N=1.8 \mathrm{~dB})$

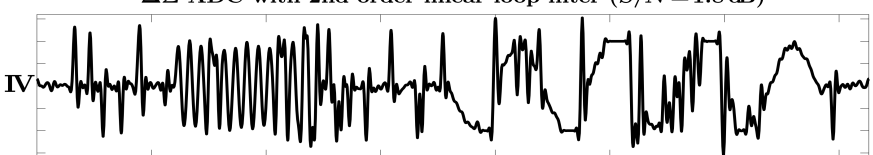

$\Delta \Sigma$ ADC with CMTF-based loop filter $(S / N=18.7 \mathrm{~dB})$

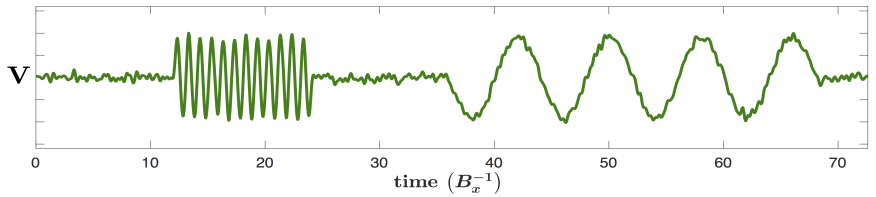

Figure 8. Comparative performance of $\Delta \Sigma$ ADCs with linear and nonlinear analog loop filters.

the filtered output is approximately $20 \mathrm{~dB}$ in the absence of the outlier noise. The amount of the outlier noise is such that the resulting signal-to-noise ratio for the filtered output of the $\Delta \Sigma$ $\mathrm{ADC}$ with a 1st-order linear loop filter is approximately $6 \mathrm{~dB}$ in the absence of the AWGN.

As one can see in panels III and IV of Fig. 8, the linear loop filters are ineffective in suppressing the impulsive noise. Further, the performance of the $\Delta \Sigma \mathrm{ADC}$ with the 2nd-order linear loop filter (see panel IV of Fig.8) is more severely degraded by high-power noise, especially by high-amplitude outlier noise such that the condition $|x(t-\Delta t)+(w * \nu)(t)|<V_{c}$ is not satisfied for all $t$. On the other hand, as can be seen in panel $\mathrm{V}$ of Fig. 8, the $\Delta \Sigma$ ADC with the CMTF-based loop filter improves the signal-to-noise ratio by about $13 \mathrm{~dB}$ in comparison with the $\Delta \Sigma \mathrm{ADC}$ with the 1st-order linear loop filter, thus removing about $95 \%$ of the impulsive noise.

More importantly, as can be seen in panel III of Fig. 9, increasing the impulsive noise power by an order of magnitude hardly affects the output of the $\Delta \Sigma$ ADC with the CMTFbased loop filter (and thus about $99.5 \%$ of the impulsive noise is removed), while further exceedingly degrading the output of the $\Delta \Sigma \mathrm{ADC}$ with the 1st-order linear loop filter (panel II).

\section{VI. $\Delta \boldsymbol{\Sigma}$ ADC WITH ADAPTIVE CMTF}

In this section we outline how to design a CMTF with an adaptive (possibly asymmetric) blanking range $\left[\alpha_{-}, \alpha_{+}\right]$. To ensure that the values of the difference signal $h_{\tau} *(x-y)$ that lie outside of $\left[\alpha_{-}, \alpha_{+}\right]$are outliers, one can identify $\left[\alpha_{-}, \alpha_{+}\right]$with Tukey's range [41], a linear combination of the 1 st $\left(Q_{1}\right)$ and the 3 rd $\left(Q_{3}\right)$ quartiles of the linear-regime

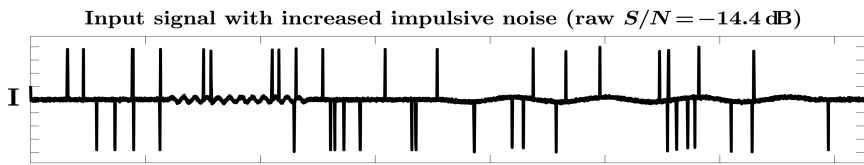

$\Delta \Sigma$ ADC with 1st-order linear loop filter $(S / N=-0.7 \mathrm{~dB})$

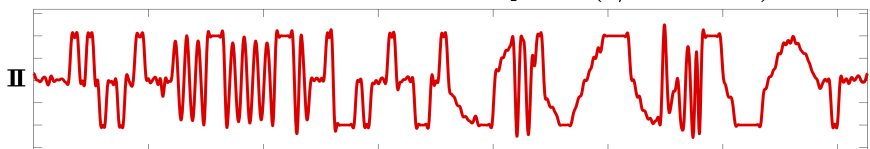

$\Delta \Sigma$ ADC with CMTF-based loop filter $(S / N=18.6 \mathrm{~dB})$

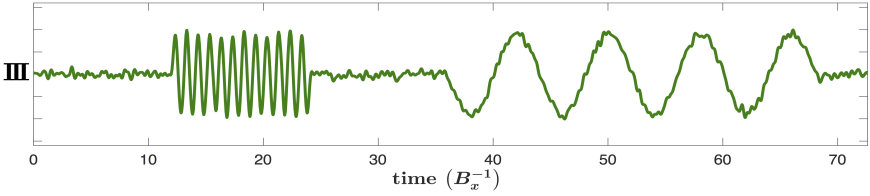

Figure 9. Resistance of $\Delta \Sigma$ ADC with CMTF-based loop filter to increase in impulsive noise.

difference signal (see [33], [34] for discussion of quantiles of continuous signals):

$$
\left[\alpha_{-}, \alpha_{+}\right]=\left[Q_{1}-\beta\left(Q_{3}-Q_{1}\right), Q_{3}+\beta\left(Q_{3}-Q_{1}\right)\right],
$$

where $\beta$ is a coefficient of order unity (e.g. $\beta=1.5$ ). From equation (20), for a symmetrical distribution the range that excludes outliers can also be obtained as $\left[\alpha_{-}, \alpha_{+}\right]=[-\alpha, \alpha]$, where $\alpha$ is given by

$$
\alpha=(1+2 \beta) Q_{2}^{\star},
$$

and where $Q_{2}^{\star}$ is the 2nd quartile (median) of the absolute value of the difference signal $\left|h_{\tau} *(x-y)\right|$.

\section{A. Median Tracking Filter}

Let $x(t)$ be a quasi-stationary signal characterized by an average crossing rate $\langle f\rangle$ of the threshold equal to the second quartile (median) of $x(t)$. (See [49], [50] for discussion of threshold crossing rates.) Let us further consider the signal $Q_{2}(t)$ related to $x(t)$ by the following differential equation:

$$
\frac{\mathrm{d}}{\mathrm{d} t} Q_{2}=\frac{A}{T} \operatorname{sgn}\left(x-Q_{2}\right),
$$

where $A$ is a constant with the same units as $x$ and $Q_{2}$, and $T$ is a constant with the units of time. According to equation (22), $Q_{2}(t)$ is a piecewise-linear signal consisting

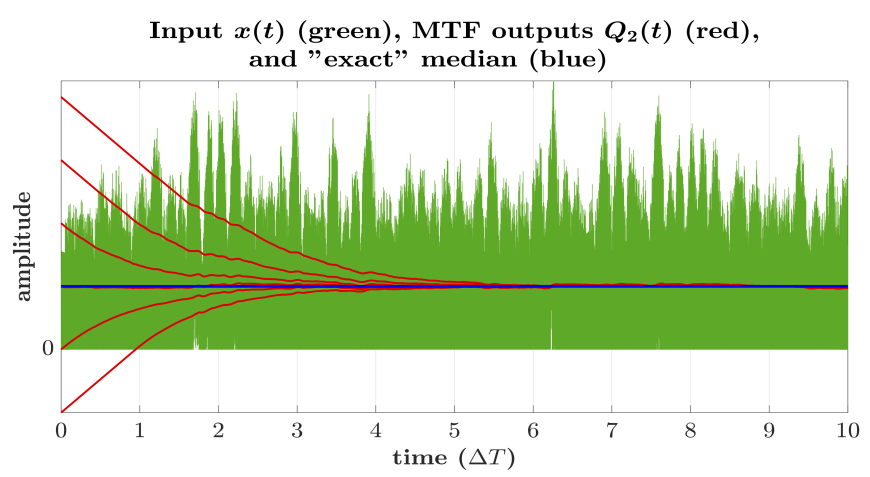

Figure 10. Illustration of MTF convergence to steady state for different initial conditions. 


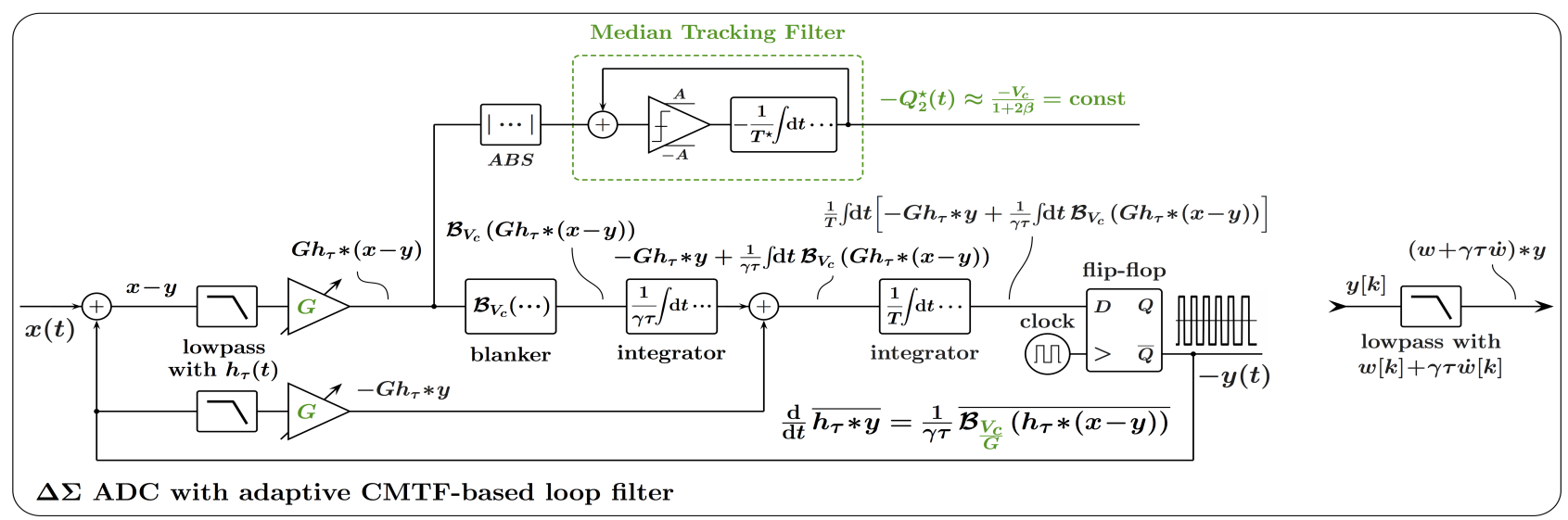

Figure 11. Outline of $\Delta \Sigma$ ADC with adaptive CMTF-based loop filter.

of alternating segments with positive $(A / T)$ and negative $(-A / T)$ slopes. Note that $Q_{2}(t) \approx$ const for a sufficiently small $A / T$ (e.g., much smaller than the product of the interquartile range and the average crossing rate $\langle f\rangle$ of $x(t)$ and its second quartile), and a steady-state solution of equation (22) can be written implicitly as

$$
\overline{\theta\left(Q_{2}-x\right)} \approx \frac{1}{2},
$$

where $\theta(x)$ is the Heaviside unit step function [47] and the overline denotes averaging over some time interval $\Delta T \gg\langle f\rangle^{-1}$. Thus $Q_{2}$ approximates the second quartile of $x(t)$ in the time interval $\Delta T$, and equation (22) describes a Median Tracking Filter (MTF). Fig. 10 illustrates the MTF's convergence to the steady state for different initial conditions.

\section{B. Adaptive CMTF}

Figure 11 provides an outline of a $\Delta \Sigma$ ADC with an adaptive CMTF-based loop filter. In this example, the 1st order lowpass filters are followed by the gain stages with the gain $G$, while the blanking value is set to $V_{c}$. Note that

$$
\mathcal{B}_{V_{c}}(G x)=G \mathcal{B}_{\frac{V_{c}}{G}}(x)
$$

and thus the "apparent" (or "equivalent") blanking value is no longer hardware limited. As shown in figure 11, the input $x(t)$ and the output $y(t)$ can be related by

$$
\frac{\mathrm{d}}{\mathrm{d} t} \overline{h_{\tau} * y}=\frac{1}{\gamma \tau} \overline{\mathcal{B}_{\frac{V_{c}}{G}}\left(h_{\tau} *(x-y)\right)} .
$$

If an automatic gain control circuit maintains a constant output $-V_{c} /(1+2 \beta)$ of the MTF circuit in Fig. 11, then the apparent blanking value $\alpha=V_{c} / G$ in equation (25) will be given by equation (21).

\section{Performance example}

1) Simulation parameters: To emulate the analog signals in the examples below, the digitization rate is two orders of magnitude higher than the sampling rate $F_{\mathrm{s}}$. The signal of interest is a Gaussian baseband signal in the nominal frequency rage $\left[0, B_{x}\right]$. It is generated as a broadband white Gaussian noise filtered with a root-raised-cosine filter with the roll-off factor $1 / 4$ and the bandwidth $5 B_{x} / 4$. The noise affecting the signal of interest is a sum of an AWGN background component and white impulsive noise $i(t)$. The impulsive noise is modeled as symmetrical (bipolar) Poisson shot noise:

$$
i(t)=\nu(t) \sum_{k=1}^{\infty} \delta\left(t-t_{k}\right)
$$

where $\nu(t)$ is AWGN noise, $t_{k}$ is the $k$-th arrival time of a Poisson process with the rate parameter $\lambda$, and $\delta(x)$ is the Dirac $\delta$-function [48]. In the examples below, $\lambda=B_{x}$. The gain $G$ is chosen to maintain the output of the MTF in Fig. 11 at $-V_{c} /(1+2 \beta)$, and the digital lowpass filter $w[k]$ is the root-raised-cosine filter with the roll-off factor $1 / 4$ and the bandwidth $5 B_{x} / 4$. The remaining hardware parameters are the same as those in Section V. Further, the magnitude of the input $x(t)$ is chosen to ensure that the average absolute value of the output signal is approximately $V_{c} / 5$.

2) Comparative channel capacities: For the simulation parameters described above, Fig. 12 compares the simulated channel capacities (calculated from the baseband SNRs using the Shannon formula [45]) for various signal+noise compositions, for the linear signal processing chain (red lines) and the CMTF-based chain of Fig. 11 (green lines) with $\beta=1.5$ (solid lines), $\beta=1.75$ (dashed), and $\beta=1.25$ (dotted).

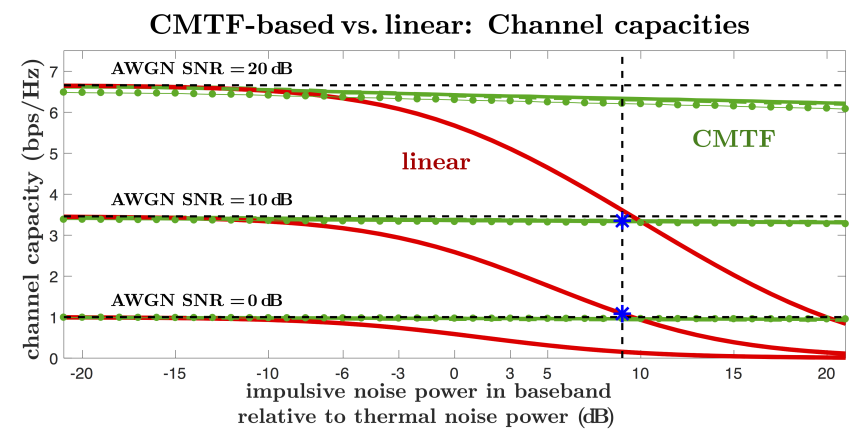

Figure 12. Comparison of simulated channel capacities for the linear processing chain (red lines) and the CMTF-based chains (green lines) with $\beta=1.75$ (solid lines), $\beta=1.5$ (dashed), and $\beta=1.25$ (dotted). The meaning of the blue asterisks is explained in the text. 
As one can see in Fig. 12 (and compare with the simplified diagram of Fig. 1), linear and the CMTF-based chains provide effectively equivalent performance when the AWGN dominates over the impulsive noise. However, the CMTFbased chains are insensitive to further increase in the impulsive noise when the latter becomes comparable or dominates over the thermal (Gaussian) noise, thus providing resistance to impulsive interference.

3) Disproportionate effect on baseband PSDs: For a mixture of white Gaussian and white impulsive noise, Fig. 13 illustrates reduction of the spectral density of impulsive noise in the signal baseband without affecting that of the signal of interest. In the figure, the black lines correspond to the "ideal" signal of interest (without noise), and the colored lines correspond to the signal+noise mixtures. The baseband power of the AWGN is one tenth of that of the signal of interest (10 dB AWGN SNR), and the baseband power of the impulsive noise is approximately 8 times $(9 \mathrm{~dB})$ that of the AWGN. The value of the parameter $\beta$ for Tukey's range is $\beta=1.5$. As can be seen in the figure, for the CMTF-based chain the baseband SNR increases from $0.5 \mathrm{~dB}$ to $9.7 \mathrm{~dB}$.

For both the linear and the CMTF-based chains the observed baseband noise can be considered to be effectively Gaussian, and we can use the Shannon formula [45] based on the achieved baseband SNRs to calculate the channel capacities. Those are marked by the blue asterisks on the respective red and solid green curves in Fig. 12.

Fig. 14 provides a similar illustration with additional interference in an adjacent channel. Such interference increases the apparent blanking value needed to maintain effectively linear CMTF behavior in the absence of the outliers, slightly reducing the effectiveness of the impulsive noise suppression. As a result, the baseband SNR increases from $0.5 \mathrm{~dB}$ to only $8.5 \mathrm{~dB}$

\section{CONCLUSION}

In this paper we propose a conceptually new approach to analog-to-digital conversion of signals affected by nonGaussian noise. It is based on incorporating nonlinear analog loop filters in $\Delta \Sigma$ ADCs in place of the typically present linear loop filters. Such an approach allows us to disproportionately affect signals with different temporal and/or amplitude structures, thus enabling reduction of the spectral density of partic-
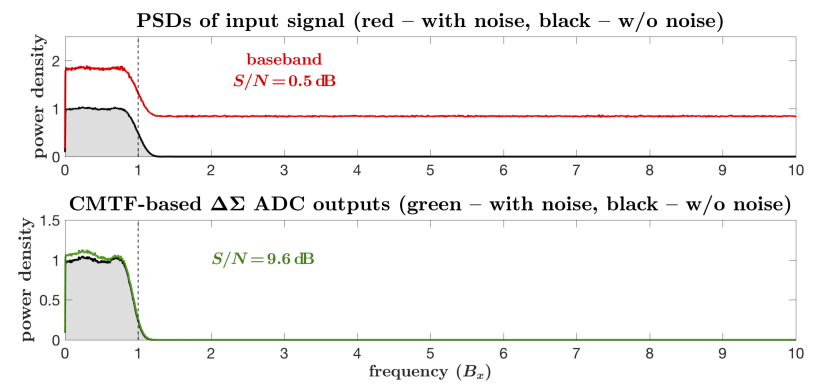

Figure 13. Illustration of reduction of the spectral density of impulsive noise in the signal baseband without affecting that of the signal of interest: Mixture of white Gaussian and white impulsive noise. ular types of non-Gaussian interferences (such as, for example, impulsive noise) in the signal passband without significantly affecting the signal of interest. This can considerably increase quality of the acquired signal over that achievable by linear filtering.

As illustrated in Sections V and VI, the $\Delta \Sigma$ ADCs with CMTF-based loop filters can be very effective in mitigating broadband impulsive noise, especially when such noise dominates over the background non-impulsive noise. Similarly, various other nonlinear modifications of the loop filters in $\Delta \Sigma$ ADCs can be constructed, tailored for specific signal and interference types and for particular applications, and for specific performance requirements. In addition, as outlined in Section VI, adaptive nonlinear loop filters can be developed to improve performance under non-stationary and timevarying signal and noise conditions. An important property of the presented approach is that, while being nonlinear in general, the proposed ADCs largely behave linearly. They exhibit nonlinear behavior only intermittently, in response to noise outliers, thus avoiding the detrimental effects, such as instabilities and intermodulation distortions, often associated with nonlinear signal processing.

Further, the temporal and/or amplitude structure (and thus the distributions) of non-Gaussian signals are generally modifiable by linear filtering, and non-Gaussian interference can often be converted from sub-Gaussian into super-Gaussian, and vice versa, by linear filtering [9], [10], [32, e.g.]. Thus the ability of the proposed $\Delta \Sigma$ ADCs with analog nonlinear loop filters to mitigate impulsive (super-Gaussian) noise can translate into mitigation of non-Gaussian noise and interference in general, including sub-Gaussian noise. For example, a linear analog filter can be employed as an input front end filter of the ADC to increase the peakedness of the interference, and the $\Delta \Sigma$ ADCs with analog nonlinear loop filter can perform analog-to-digital conversion combined with mitigation of this interference. Subsequently, if needed, a digital filter can be employed to compensate for the impact of the front end filter on the signal of interest.

\section{ACKNOWLEDGMENTS}

The authors would like to thank Arlie Stonestreet II and Kyle D. Tidball of Ultra Electronics ICE, Manhattan, KS, USA, for their valuable suggestions and input.
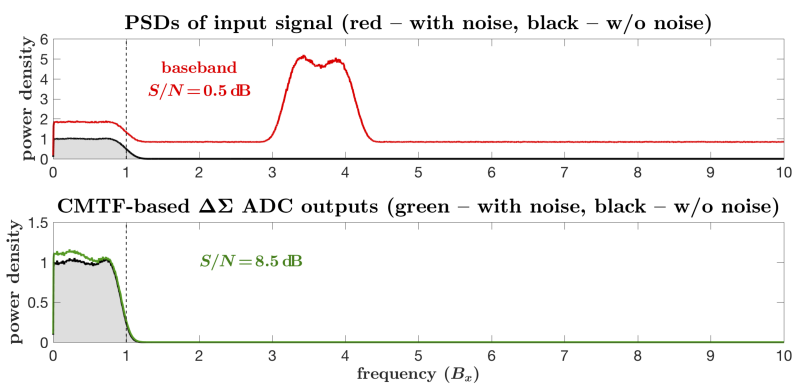

Figure 14. Illustration of reduction of the spectral density of impulsive noise in the signal baseband without affecting that of the signal of interest: Example of figure 13 with additional interference in adjacent channel. 


\section{REFERENCES}

[1] M. Stojanovic and J. Preisig, "Underwater acoustic communication channels: Propagation models and statistical characterization," IEEE Commun. Mag., vol. 47, no. 1, pp. 84-89, January 2009.

[2] J. S. G. Panaro, F. R. B. Lopes, L. M. Barreira, and F. E. Souza, "Underwater acoustic noise model for shallow water communications," in XXX Simpósio Brasileiro de Telecomunicações (SBrT 2012), Brazil, 13-16 September 2012.

[3] G. B. Kinda, Y. Simard, C. Gervaise, J. I. Mars, and L. Fortier, "Arctic underwater noise transients from sea ice deformation: Characteristics, annual time series, and forcing in Beaufort Sea," J. Acoust. Soc. Am., vol. 138, no. 4, pp. 2034-2045, Oct. 2015.

[4] J. D. Parsons, The Mobile Radio Propagation Channel, 2nd ed. Chichester: Wiley, 2000.

[5] X. Yang and A. P. Petropulu, "Co-channel interference modeling and analysis in a Poisson field of interferers in wireless communications," IEEE Trans. Signal Process., vol. 51, no. 1, pp. 64-76, 2003.

[6] A. V. Nikitin, "On the impulsive nature of interchannel interference in digital communication systems," in Proc. IEEE Radio and Wireless Symposium, Phoenix, AZ, 2011, pp. 118-121.

[7] _ , "On the interchannel interference in digital communication systems, its impulsive nature, and its mitigation," EURASIP J. Adv. Signal Process., vol. 2011, no. 137, 2011.

[8] A. V. Nikitin, M. Epard, J. B. Lancaster, R. L. Lutes, and E. A. Shumaker, "Impulsive interference in communication channels and its mitigation by SPART and other nonlinear filters," EURASIP J. Adv. Signal Process., vol. 2012, no. 79, 2012.

[9] A. V. Nikitin, R. L. Davidchack, and T. J. Sobering, "Adaptive analog nonlinear algorithms and circuits for improving signal quality in the presence of technogenic interference," in Proceedings of IEEE Military Communications Conference 2013, San Diego, CA, 18-20 Nov. 2013.

[10] A. V. Nikitin, R. L. Davidchack, and J. E. Smith, "Out-of-band and adjacent-channel interference reduction by analog nonlinear filters," EURASIP J. Adv. Signal Process., vol. 2015, no. 12, 2015.

[11] J. Carey, "Noise wars: Projected capacitance strikes back against internal noise," $E D N$, pp. 61-65, Jan. 19, 2012.

[12] T. B. Gabrielson, "Mechanical-thermal noise in micromachined acoustic and vibration sensors," IEEE Trans. Electron Devices, vol. 40, no. 5, pp. 903- 909, 1993

[13] F. Mohd-Yasin, D. J. Nagel, and C. E. Korman, "Noise in MEMS," Meas. Sci. Technol., vol. 21, no. 1, pp. 1-22, 2010.

[14] S. H. Ardalan and J. J. Paulos, "An analysis of nonlinear behavior in delta-sigma modulators," IEEE Trans. Circuits Syst., vol. 34, no. 6, pp. 593-603, 1987.

[15] E. Janssen and A. van Roermund, Look-Ahead Based Sigma-Delta Modulation. Springer, 2011

[16] A. Chopra, "Modeling and mitigation of interference in wireless receivers with multiple antennae," $\mathrm{PhD}$ thesis, The University of Texas at Austin, December 2011.

[17] M. Walessa and M. Datcu, "Model-based despeckling and information extraction from SAR images," IEEE Trans. Geosci. Remote Sens., vol. 38 , no. 5 , pp. $2258-2269,2000$.

[18] R. Dragomir, S. Puscoci, and D. Dragomir, "A synthetic impulse noise environment for DSL access networks," in Proceedings of the 2nd International conference on Circuits, Systems, Control, Signals (CSCS'11), 2011, pp. 116-119.

[19] V. Guillet, G. Lamarque, P. Ravier, and C. Léger, "Improving the power line communication signal-to-noise ratio during a resistive load commutation," J. Commun., vol. 4, no. 2, pp. 126-132, 2009.

[20] M. Katayama, T. Yamazato, and H. Okada, "A mathematical model of noise in narrowband power line communication systems," IEEE J. Sel. Areas Commun., vol. 24, no. 7, pp. 1267-1276, 2006.

[21] M. Nassar, J. Lin, Y. Mortazavi, A. Dabak, I. H. Kim, and B. L. Evans, "Local utility power line communications in the $3-500 \mathrm{kHz}$ band: Channel impairments, noise, and standards," IEEE Signal Process. Mag., vol. 29, no. 5, pp. 116-127, 2012.

[22] M. Nassar, A. Dabak, I. H. Kim, T. Pande, and B. L. Evans, "Cyclostationary noise modeling in narrowband powerline communication for Smart Grid applications," in 2012 IEEE Int. Conf. Acoust. Speech Signal Process. (ICASSP), 25-30 March 2012, pp. 3089-3092.

[23] J. Lin, M. Nassar, and B. L. Evans, "Impulsive noise mitigation in powerline communications using sparse Bayesian learning," IEEE J. Sel. Areas Commun., vol. 31, no. 7, pp. 1172-1183, 2013.
[24] A. V. Nikitin, D. Scutti, B. Natarajan, and R. L. Davidchack, "Blind adaptive analog nonlinear filters for noise mitigation in powerline communication systems," in Proc. IEEE International Symposium on Power Line Communications and Its Applications (ISPLC 2015), Austin, TX, 29-31 March 2015

[25] S. A. Bhatti, Q. Shan, R. Atkinson, M. Vieira, and I. A. Glover, "Vulnerability of Zigbee to impulsive noise in electricity substations," in General Assembly and Scientific Symposium, 2011 XXXth URSI, 13-20 Aug. 2011.

[26] S. R. Mallipeddy and R. S. Kshetrimayum, "Impact of UWB interference on IEEE 802.11a WLAN system," in National Conference on Communications (NCC), 2010.

[27] C. Fischer, "Analysis of cellular CDMA systems under UWB interference," in International Zurich Seminar on Communications, 2006, pp. $130-133$.

[28] K. Slattery and H. Skinner, Platform Interference in Wireless Systems. Elsevier, 2008.

[29] F. Leferink, F. Silva, J. Catrysse, S. Batterman, V. Beauvois, and A. Roc'h, "Man-made noise in our living environments," Radio Science Bulletin, no. 334, pp. 49-57, September 2010.

[30] G. R. Arce, N. A. Grabowski, and N. C. Gallagher, "Weighted median filters with sigma-delta modulation encoding," IEEE Trans. Signal Process., vol. 48, no. 2, pp. 489-498, 2000.

[31] Z. Miao and X. Jiang, "Weighted iterative truncated mean filter," IEEE Trans. Signal Process., vol. 61, no. 16, pp. 4149-4160, 2013.

[32] A. V. Nikitin, "Method and apparatus for signal filtering and for improving properties of electronic devices," US patents 8,489,666 (16 July 2013), 8,990,284 (24 March 2015), 9,117,099 (August 25, 2015), and 9,467,113 (11 October 2016)

[33] A. V. Nikitin and R. L. Davidchack, "Signal analysis through analog representation," Proc. R. Soc. Lond. A, vol. 459, no. 2033, pp. 11711192, 2003.

[34] — , "Adaptive approximation of feedback rank filters for continuous signals," Signal Processing, vol. 84, no. 4, pp. 805-811, 2004

[35] - "Method and apparatus for analysis of variables," Patent, US Patents 7,133,568 (Nov. 7, 2006) and 7,242,808 (July 10, 2007).

[36] A. V. Nikitin, "Method and apparatus for real-time signal conditioning, processing, analysis, quantification, comparison, and control," Patent, US Patents 7,107,306 (Sep. 12, 2006), 7,418,469 (Aug. 26, 2008), and 7,617,270 (Nov. 10, 2009).

[37] — "Method and apparatus for adaptive real-time signal conditioning and analysis," Patent, US Patent 8,694,273 (April 8, 2014).

[38] G. I. Bourdopoulos, A. Pnevmatikakis, V. Anastassopoulos, and T. L. Deliyannis, Delta-Sigma Modulators: Modeling, Design and Applications. London: Imperial College Press, 2003.

[39] W. Kester, Ed., Data Conversion Handbook. Oxford: Elsevier, 2005.

[40] Y. Geerts, M. Steyaert, and W. M. C. Sansen, Design of Multi-Bit Delta-Sigma A/D Converters, ser. The Springer International Series in Engineering and Computer Science. Springer US, 2006.

[41] J. W. Tukey, Exploratory Data Analysis. Addison-Wesley, 1977.

[42] P. J. Huber, Robust Statistics, ser. Wiley Series in Probability and Statistics. Wiley, 2005.

[43] S. V. Vaseghi, Advanced Digital Signal Processing and Noise Reduction, 4th ed. Wiley, 2008.

[44] A. V. Nikitin, "Pulse pileup effects in counting detectors," PhD thesis, University of Kansas, Lawrence, 1998

[45] C. E. Shannon, "Communication in the presence of noise," Proc. Institute of Radio Engineers, vol. 37, no. 1, pp. 10-21, Jan. 1949.

[46] I. N. Bronshtein and K. A. Semendiaev, Handbook of Mathematics, 3rd ed. Springer, 1997.

[47] R. Bracewell, The Fourier Transform and Its Applications, 3rd ed. New York: McGraw-Hill, 2000, ch. "Heaviside's Unit Step Function, $H(x)$ ", pp. 61-65.

[48] P. A. M. Dirac, The Principles of Quantum Mechanics, 4th ed. London: Oxford University Press, 1958.

[49] S. O. Rice, "Mathematical analysis of random noise," Bell System Technical Journal, vol. 23, pp. 282-332, 1944, Ibid. 24:46-156, 1945. Reprinted in: Nelson Wax, editor, Selected papers on noise and stochastic processes. Dover, New York, 1954.

[50] A. V. Nikitin, R. L. Davidchack, and T. P. Armstrong, "The effect of pulse pile-up on threshold crossing rates in a system with a known impulse response," Nucl. Instr. \& Meth., vol. A411, pp. 159-171, 1998. 